\title{
Presentación
}

\section{Orden social más allá del Estado}

La ausencia o presencia precaria del Estado en espacios marginalizados y periféricos es comúnmente asociada con el colapso del orden social, el surgimiento de economías ilegales y el aumento de la violencia. Sin embargo, una creciente agenda de investigación ha mostrado cómo la construcción del orden social es posible con Estados "débiles" o inexistentes, donde actores no estatales y estatales establecen normas que regulan efectivamente el comportamiento de la población, cuestionando así una visión excesivamente Estado-céntrica del orden social. Al mismo tiempo, la existencia de estas diversas formas de orden requiere que exploremos la naturaleza de los vínculos entre actores estatales y no estatales; las percepciones e imaginarios sobre estos espacios; las percepciones e imaginarios de sus habitantes sobre el Estado, y la forma como la violencia puede ser un elemento característico de estos órdenes sociales.

En este número de Revista CS contamos con un excelente grupo de artículos que contribuyen a este vibrante debate internacional, explorando estos temas desde diferentes perspectivas metodológicas y conceptuales: desde un artículo que compara casos en diferentes regiones del mundo hasta exploraciones etnográficas de comunidades marginalizadas en Colombia, pasando por la construcción de orden social en prisiones bonaerenses y un análisis de la política pública de seguridad en Chile. Esta selección es un reflejo de la diversidad de la producción intelectual de esta agenda de investigación que esperábamos encontrar cuando se lanzó la convocatoria de la revista.

El primer artículo, títulado "Estado, ilegalidad y orden social en perspectiva comparativa: una aproximación mediante estudios de caso", de Daniel Míguez, nos ofrece importantes reflexiones sobre la articulación entre legalidad e ilegalidad en la construcción de órdenes sociales. Comúnmente, este vínculo se ha estudiado en países periféricos donde el Estado tiene una limitada capacidad de imponer el orden legal formal y no logra penetrar efectivamente todo su territorio. El supuesto de que la construcción de órdenes sociales por la interacción de actores legales e ilegales solo tiene lugar en estos países, y no en Estados "consolidados", lleva, como bien argumenta el autor, no solo a la "exotización" de la periferia, sino también a 
un déficit de conocimiento acerca de estas relaciones en contextos de alto grado de estatalidad. No porque estos vínculos sean menos visibles en los países del norte global, debemos asumir que no existen. A través de la exploración de casos en Rusia, América Latina y Estados Unidos, el autor presenta posibles prototipos para describir la articulación entre legalidad e ilegalidad, mostrando que estos vínculos no son propiedad exclusiva de los países "periféricos". Adicionalmente, el contraste de estos casos le permite al autor derivar una hipótesis sugestiva sobre el papel de la flagrancia de estos vínculos para la legitimidad de los órdenes sociales legales. La mayor visibilidad de la articulación de legalidad e ilegalidad en países con Estados más débiles puede reducir la legitimidad de las normas formales-legales. Esta relación entre, de un lado, la visibilidad de los nexos entre legalidad e ilegalidad, y, de otro lado, la legitimidad de los órdenes formales-legales e ilegales debe, sin ninguna duda, ser explorada más sistemáticamente en futuras investigaciones.

Frecuentemente se piensa que la construcción de orden estatal en las periferias urbanas es una condición necesaria para reducir los niveles de violencia y la influencia de grupos criminales. Si bien la construcción de estos órdenes estatales en las periferias implica una mayor presencia del Estado, es también importante explorar las percepciones y evaluaciones de los habitantes de barrios urbanos periféricos sobre la acción estatal. En su análisis sobre las políticas de seguridad pública en barrios urbano-marginales de Chile, el artículo "Violencia y seguridad en los márgenes urbanos: la respuesta chilena en los vecindarios (2001-2019)", de Alejandra Luneke y Fernanda Varela, nos muestra cómo -a pesar de que las políticas incluyen componentes de participación comunitaria y fortalecimiento de la presencia estatal- los habitantes de las comunidades intervenidas expresan desconocer estas iniciativas públicas o no tienen una buena evaluación de ellas.

Aunque los barrios urbanos marginalizados son conceptualizados con frecuencia como zonas "desprovistas" de Estado y, consecuentemente, con altos niveles de violencia, es necesario repensar la presencia estatal en estos territorios. Basado en su etnografía del distrito de Aguablanca (Cali, Colombia), Carlos Andrés Arana-Castañeda sugiere cómo la simultánea presencia y ausencia del Estado en esta zona constituye un forma de orden social que permite la reproducción de una violencia racializada. La acción estatal-por ejemplo, a través de la construcción de un proyecto de vivienda-choca con procesos de organización y gestión comunitaria, provacando el rechazo y la protesta de la comunidad. En este sentido, es pertinente pensar la acción estatal y la presencia del Estado en las zonas marginalizadas y comprender cómo esta responde a la construcción y preservación de órdenes sociales en la ciudad (como un todo) que buscan mantener la marginalización de los habitantes de estas zonas. 
Continuando con esta perspectiva que busca comprender el orden social en territorios periféricos y racializados, el artículo "Orden social y violencia en Buenaventura: entre el outsourcing criminal y la construcción de paz desde abajo", de Lizeth Sinisterra-Ossa e Inge Helena Valencia, analiza la reconfiguración de órdenes sociales en el contexto de posacuerdo y transición que vive Colombia tomando como caso Buenaventura. Las autoras indagan sobre las formas como los actores armados ilegales regulan la vida social en este lugar impactado por las lógicas del conflicto armado y del narcotráfico, y las maneras como administran y utilizan la violencia. $\mathrm{Al} \mathrm{mismo} \mathrm{tiempo,} \mathrm{muestran} \mathrm{las} \mathrm{estrategias} \mathrm{de} \mathrm{negociación} \mathrm{y} \mathrm{resistencia} \mathrm{por} \mathrm{parte}$ de colectivos de mujeres negras, estrategias que pueden ser comprendidas como formas de construcción de paz y agencia afrofemenina en espacios racializados.

Finalmente, cerramos la parte temática de este número con el artículo "Entre la redención y la ruta de la inclusión. Intervenciones tratamentales de actores no estatales en cárceles bonaerenses", el cual propone pensar el orden social construido en las cárceles de la Provincia de Buenos Aires, a través de la relación entre actores estatales y no estatales. En este artículo, las autoras (Andrea Natalia Lombraña, Natalia Soledad Ojeda, Carolina Emilia Di Próspero y María Belén Pepe) identifican los repertorios de acción de las intervenciones hechas por organizaciones de la sociedad civil para atender los procesos de resocialización de la población carcelaria. Las autoras buscan evidenciar las rupturas y negociaciones entre actores estatales y no estatales en torno a las propuestas de resocialización de las personas privadas de la libertad.

Esta selección de artículos nos muestra la pertinencia de comprender otras formas de orden social que se configuran "más allá del Estado", en donde actores no estatales, ligados a la violencia y al crimen organizado, pero también organizaciones civiles de distinta naturaleza, intentan regular la vida social y política de la población. Así mismo, pensar el orden social es una invitación a entender los retos que enfrentan los Estados hoy en día, en un contexto de crisis institucional y polarización, donde la resolución de conflictos y la administración de justicia se revelan como agendas urgentes de la investigación social y la intervención pública.

Inge Helena Valencia (Universidad Icesi, Colombia)

Juan Albarracín (Universidad Icesi, Colombia)

\section{Editores invitados}

